Vol. 44, N. 2 : pp. 155 - 163, June, 2001

ISSN 1516-8913 Printed in Brazil

\title{
Natural Regeneration in a Quaternary Coastal Plain in Southern Brazilian Atlantic Rain Forest
}

\author{
Cleber Ibraim Salimon ${ }^{1 *}$ and Raquel R. B. Negrelle \\ Lab. Oikos, Dep. Botânica, Universidade Federal do Paraná. Cx.Postal 19031. CEP 81531-970 Curitiba - PR, \\ Brazil
}

\begin{abstract}
Composition, structure and dynamics of an eight year old secondary forest was studied at Reserva Volta Velha (26 $04^{\prime}$ 'S; 48 38'W), southern Brazil. A 0.72ha plot was divided into 36 subplots of 20X10m, where all trees/shrubs greater than $1 \mathrm{~m}$ tall were identified, measured (height/diameter) and evaluated (successional status). The results were: (1) 95 species collected within 68 genera and 44 families; the most species rich families were Myrtaceae and Asteraceae with 8 species each; (2) the most important species (considering biomass and density) were Psidium cattleianum, Eupatorium casarettoi, Ocotea pulchella and Ternstroemia brasiliensis; (3) the most similar area was a fallow abandoned 35 years ago; (4) the higher species diversity were found in border subplots, indicating that most of the species do not tolerate extreme conditions in the center of the opening, and are colonizing the area through the borders.
\end{abstract}

Key words: Atlantic Rain Forest, floristics, structure, regeneration, dynamics

\section{INTRODUCTION}

After centuries of land cover change by man's activity on Earth, many researchers have turned their efforts towards a better understanding of the composition and function of natural ecosystems, and especially the processes related to regeneration of natural and degraded areas (Gomes-Pompa et al, 1991). Accordingly, it is common knowledge that through natural regeneration, many ecosystems can "recover". This recovery is dependent on some posdisturbance environmental restraints, such as soil characteristics, i.e. density and chemistry, climate (temperature and humidity), light, proximity to sources of diaspores, and the existence of an active soil seed bank (Denslow, 1987; Garwood, 1989).
The Brazilian Atlantic Forest fits well into the context of anthropogenic land cover change. Originally, this phytogeographic unit extended from northeastern to southern Brazilian Coast. Due to climatic changes during the glacial and interglacial periods in the Quaternary (Por, 1992; Ab'Saber, 1979), this vegetation showed some discontinuity even before the arrival of the Europeans at the Brazilian Coast. Now, after almost 500 years from the first European colonization, this vegetation is only about 5\% $\left(20,000 \mathrm{~km}^{2}\right)$ of the original area (Por, 1992).

Most of the remnants of the Atlantic Rain Forest are in areas of difficult access, such as steep hills and mountainous areas. The lowlands, especially those in the southeastern and southern Brazil are vanishing due to real estate expansion and exploitation. The study of these communities in

\footnotetext{
* Author for correspondence
} 
different successional states is crucial to our understanding of their natural regeneration, in order to accumulate enough knowledge to reestablish the degraded areas.

The goal of this study is to contribute to the understanding of the natural regeneration dynamics of the Atlantic Forest, through the comparative analysis of the floristic composition and structure of a fallow of approximately 8 years old in a private reserve in Itapoá, SC, Brazil.

Our major questions were 1 . In which way this studied area differs from other adjacent areas in more advanced successional stages? 2. Floristically, which are the similarities comparing the studied area with other coastal lowland forest ecosystems? 3. Based on the species successional status and increasing of diversity, how is succession taking place?

\section{METHODS}

Study Area: The Reserva Volta Velha $\left(26^{\circ} 04^{\prime} \mathrm{S}\right.$; $48^{\circ} 38^{\prime}$ W; Figure 1) in the Itapoá county, State of Santa Catarina, Brazil, is a private reserve (RPPN), and is part of the area considered as the Atlantic Forest Biosphere Reserve.

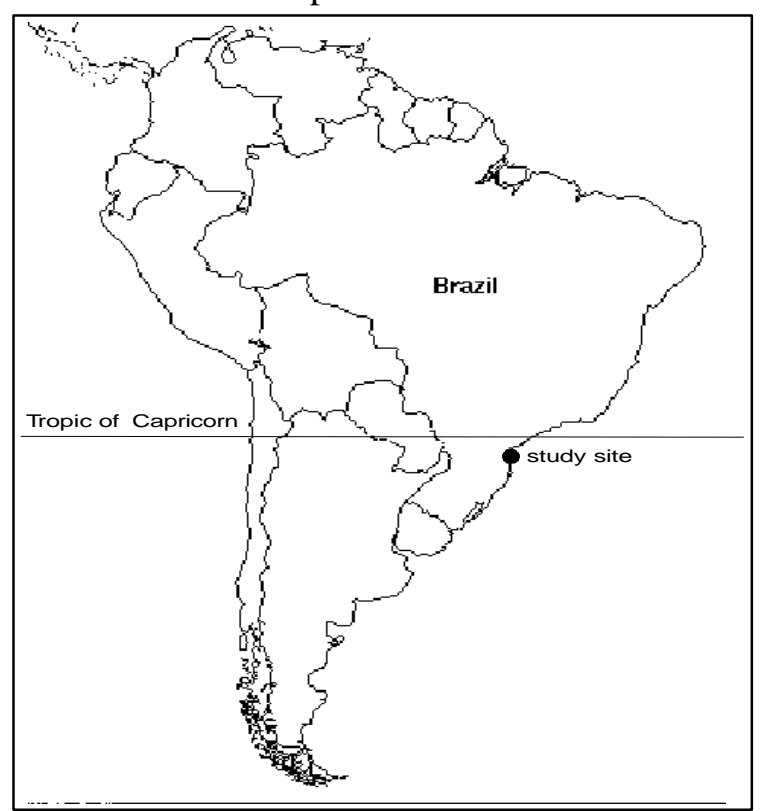

Figure 1 - Location of the study area of a 8-year-old fallow in Tropical Rain Forest, Santa Catarina, Southern Brazil.

The regional climate is classified as AB'3ra', according to Thornthwaite's classification; which means superhumid, mesothermic, with little or no hydrological deficit and annual potential summer evapotranspiration lower than $48 \%$ (Santa Catarina, 1986). The mean annual temperature in this zone is $21.4{ }^{\circ} \mathrm{C}$. The hottest month is January, with a maximum temperature of $27^{\circ} \mathrm{C}$ and the coldest month is June with minimum temperature of $16{ }^{\circ} \mathrm{C}$. Mean annual rainfall is $2170 \mathrm{~mm}$, with no well-defined dry period. Maximum rainfall occurs between January and March. Therefore, although the reserve is geographically outside the tropics, this region can be climatically considered tropical (Klein, 1978; Negrelle, 1995).

The specific study site is located at a hectare that was cut down for manihot plantation. After two years of cultivation and at least two fires, it was abandoned eight years before the beginning of this study. This site was surrounded by secondary forest (previously cultivated and abandoned 35 years ago) and also by forest with no historical or visual signs of perturbation - henceforth referred to as "intact forest". This site is dominated by plains with little altimetric variations - mean topographic levels at $0.033 \%$ - with sandy soils classified as Podzol, according to Dorneles (1996) and Lolis (1996).

Floristic survey: The floristic composition was studied by collecting all vascular plants found within the one hectare from Dec/95 to Dec/96. The material collected was identified following classical procedures. All flowering identified material was registered at the Herbarium of the Universidade Federal do Paraná (UPCB). Vegetative material was identified by comparison but not registered. Subspecies and/or varieties were not considered in the present paper.

Community Structure: Thirty-six contiguous plots $(20 \times 10 \mathrm{~m}$ each) were used for this structural analysis, making up 0.72 ha. In this survey, all trees and shrubs equal to or greater than $1 \mathrm{~m}$ tall were included, identified and collected when necessary. These individuals were marked with aluminum tags and numbered. The following measurements were taken: height - by using a rod and metric steel tapes; diameter at $15 \mathrm{~cm}$ from the soil - using a caliper or a diametric tape.

With the collected data, we analyzed the following parameters: density (D), frequency $(\mathrm{F})$, dominance (DO) and importance value (IV) (MuellerDumbois \& Ellemberg, 1974).

The diversity and floristic composition of the present study were analyzed comparatively with 3 other studies carried at the same Reserve but at stands of different successional stages; Dorneles (1996), who studied the natural 
regeneration process by analyzing the structure and composition of the seedling/sapling stratum of a 35 years old fallow; (2) Lolis (1996), who studied the structure of the tree and shrub components of the same 35 years old fallow and (3) Negrelle (1995) who studied the floristic composition and the community structure in an intact-forested area. The Shannon index was used to calculate diversity and the Sørensen's index to evaluate floristic similarity, following the procedure described in Magurran (1984).

Succession: All identified species were evaluated in order to be classified in a ecological group successional status. The classification was based on the criteria presented in Denslow (1980) and also considers the presence and absence of each species in 4 different sites, representing different successional stages (present study; Lolis, 1996; Dorneles, 1996; Negrelle, 1995). Additional autoecology information from literature were also used to establish the following categories: (1) pioneers (big gap specialists) - which germinate, grow and reproduce only in clear areas; (2) anthropogenic pioneers - which can be found as seedlings and saplings in natural conditions under the canopy of intact forests, but are especially abundant and dominant in anthropogenic disturbed areas (Kageyama, pers.comm); (3) opportunists (small gap specialists) - which can germinate in the shade, but need a clearing to grow and reproduce and (4) shade tolerants - which germinate, grow and reproduce under the canopy. The density and diversity of different successional categories were used to evaluate how succession is taking place in the studied area.

\section{RESULTS}

Floristic and structure: ninety six species of vascular plants, included in 69 genera and 45 families were collected during the floristic survey in the studied area. Among these, eight were identified only at the genus level, 6 at the family level and one as a morphospecies. The most speciouse families were Myrtaceae (8 $\mathrm{sp}$ ), Asteraceae (8 sp), Melastomataceae (7) and Rubiaceae (6). The most speciouse genera were Ilex (Aquifoliaceae); Eupatorium (Asteraceae); Ocotea (Lauraceae), Miconia (Melastomataceae);
Gomidesia (Myrtaceae) and Ossaea (Melastomataceae) (Table 1).

Among the 55 species sampled at the structural census, Psidium cattleianum, Eupatorium casarettoi, Ocotea pulchella, Ternstroemia brasiliensis, and Erythroxilon amplifolium were those with higher structural importance values. These species dominate the landscape due to the combination of higher values of density and frequency (especially $E$. cassaretoi and $\mathrm{O}$. pulchella) or basal area (especially $P$. cattleianum). (Table 2).

The Shannon's Index (H') was 1.75, a lower value than the ones found by Lolis (1996) and Negrelle (1995), $H^{\prime}=2.87$ and $H^{\prime}=3.85$, respectively (difference between Lolis (1996) and the present study was not significant $[t$ test, $\mathrm{P}<0.05])$. The greatest similarity was found for an adjacent fallow of 35 years old (Table 3 ).

P. cattleianum, O. pulchella, T. brasiliensis, E. casarettoi and Andira anthelminthica also occur in Restinga vegetation (Silva, 1990; Sugiyama and Mantovani, 1993); which are responsible - among other species - to the considerable similarity with this type of vegetation.

Other areas, such as an adjacent intact forest (Negrelle, 1995), a distant intact forest (Silva, 1985) and a very distant fallow (Torezan, 1995) had low similarity with the one we studied.

Succession: $P$. cattleianum, $O$. pulchella and $T$. brasiliensis - all among the 10 most important (IV) species - were included as anthropogenic pioneers. E. casarettoi, the second in IV, was included as a pioneer. The group with the most number of species was the opportunists (small gap specialists), with 20 species, followed by pioneers with 12 . Seven were included as shade tolerants and 10 were not included in any category due to lack of information. The assignment of an ecological group for each species is available in Table 2.

From all the species sampled in this approach, $42 \%$ were only found in plots adjacent to the older fallow or the intact forest. The plots closer to the edge were the ones with the highest diversity (Kruskal-Wallis Test. $\mathrm{P}<0.05$; Figure 2). Even though not all plots on the edge of the sampled area were close to the border of the forested region. 
Table 1 - Vascular plants occurring at a 8 year old fallow at the Atlantic Rain Forest, in Southern Brazil; where $\mathrm{H}=$ herbaceous; T = trees; L = lianas; $\mathrm{E}=$ epiphytes; $\mathrm{S}=$ shrubs; UPCB = Universidade Federal do Paraná's Herbarium, Hab - habit.

\begin{tabular}{|c|c|c|c|c|c|}
\hline FAMILY & $\begin{array}{r}\text { Voucher no. at } \\
\text { UPCB }\end{array}$ & $\begin{array}{r}\mathrm{Hab} \\
\text { it }\end{array}$ & FAMILY & $\begin{array}{r}\text { Voucher no. at } \\
\text { UPCB }\end{array}$ & $\begin{array}{r}\mathrm{Hab} \\
\text { it }\end{array}$ \\
\hline AMARYLLIDACEAE & & & MALPIGHIACEAE & & \\
\hline Hippeastrum $\mathrm{sp}$ & $* *$ & $\mathrm{H}$ & Byrsonima lingustrifolia Juss. & 28913 & $\mathrm{~T}$ \\
\hline ANNONACEAE & $* *$ & & MELASTOMATACEAE & & \\
\hline Guatteria australis A.St.Hil. & 21350 & $\mathrm{~T}$ & Miconia cinerascens Miq. & 21390 & $\mathrm{~T}$ \\
\hline Xylopia cf brasiliensis Spr. & 21354 & $\mathrm{~T}$ & Miconia rigidiuscula Cogn. & 28920 & $\mathrm{~T}$ \\
\hline AQUIFOLIACEAE & & & Miconia sellowiana Naudin & 21392 & $\mathrm{~T}$ \\
\hline Ilex pseudobuxus Reiss. & 21377 & $\mathrm{~T}$ & Ossaea amygdaloides Triana & ** & $\mathrm{S}$ \\
\hline Ilex integerrima Reiss. & 21382 & $\mathrm{~T}$ & Ossaea brachystachya Triana & $* *$ & $\mathrm{~S}$ \\
\hline Ilex theezans Mart. & 21374 & $\mathrm{~T}$ & Ossaea confertiflora (DC.) Triana & 21395 & $\mathrm{~S}$ \\
\hline Ilex $\mathrm{sp}$ & $* *$ & $\mathrm{~T}$ & Tibouchina clavata Wurdack & 21399 & $\mathrm{~S}$ \\
\hline APOCYNACEAE & & & MELIACEAE & & \\
\hline $\begin{array}{l}\text { Temnadenia stellaris (Lindl.) Miers. } \\
\text { ARACEAE }\end{array}$ & 28883 & $\mathrm{~L}$ & $\begin{array}{l}\text { Cabralea canjerana (Vell.) Mart. } \\
\text { MORACEAE }\end{array}$ & 20922 & $\mathrm{~T}$ \\
\hline $\begin{array}{l}\text { Philodendron sp. } \\
\text { ARECACEAE }\end{array}$ & $* *$ & $\mathrm{E}$ & $\begin{array}{l}\text { Ficus organensis } \text { Miq. } \\
\text { MYRSINACEAE }\end{array}$ & $* *$ & $\mathrm{~T}$ \\
\hline Attalea dubia (Mart.) Burret & ** & $\mathrm{T}$ & Myrsine coriacea (Sw.) R. Br. ex Roem. \& Schult. & 28945 & $\mathrm{~T}$ \\
\hline Geonoma schottiana Mart. & 20914 & $\mathrm{~S}$ & Myrsine venosa DC. & 21216 & $\mathrm{~T}$ \\
\hline Syagrus romanzoffiana (Cham.) Glassm. & ** & $\mathrm{T}$ & MYRTACEAE & & \\
\hline ASTERACEAE & & & Eugenia umbelliflora O.Berg. & $* *$ & $\mathrm{~T}$ \\
\hline Baccharis cassinefolia $\mathrm{L}$. & ** & $\mathrm{S}$ & Gomidesia affinis (Camb.) Legrand & 28950 & $\mathrm{~T}$ \\
\hline Elephantopus mollis Kunth & 28890 & $\mathrm{H}$ & Gomidesia fenzliana O.Berg. & 28951 & $\mathrm{~T} / \mathrm{S}$ \\
\hline Eupatorium casarettoi Steyerm. & 28891 & $\mathrm{~S}$ & Gomidesia palustris (DC.) Legrand & 21469 & $\mathrm{~T}$ \\
\hline Eupatorium inulaefolium $\mathrm{HBK}$ & $* *$ & $\mathrm{~S}$ & Myrcia multiflora (Lam.) DC. & 23912 & $\mathrm{~T}$ \\
\hline Eupatorium laevigatum Lam. & $* *$ & $\mathrm{H}$ & Myrtaceae 1 & ** & --- \\
\hline Pterocaulon lorentzii Malme & ** & $\mathrm{H}$ & Myrtaceae 2 & ** & $\mathrm{T} / \mathrm{S}$ \\
\hline Mikania trinervis Hooker et Arnott & 28892 & $\mathrm{H}$ & Psidium cattleianum Sab & 21461 & $\mathrm{~T} / \mathrm{S}$ \\
\hline Vernonia beyrichii Less. & 28893 & $\mathrm{~L}$ & NYCTAGINACEAE & & \\
\hline BIGNONIACEAE & & & Guapira asperula (Standley) Lundell & 23446 & $\mathrm{~T}$ \\
\hline Bignoniaceae sp & ** & $\mathrm{L}$ & Guapira opposita (Vell.) Reitz & 23449 & $\mathrm{~T}$ \\
\hline Jacaranda cf puberula Cham. & $* *$ & $\mathrm{~T} / \mathrm{S}$ & ORCHIDACEAE & & \\
\hline Tabebuia chrysotricha (Mart. ex DC.) & $* *$ & $\mathrm{~T}$ & Cleistes paranaensis Schltr. & 28956 & $\mathrm{H}$ \\
\hline BLECHNACEAE & & & Cyrtopodium paranaensis Schltr. & $* *$ & $\mathrm{H}$ \\
\hline Blechnum serrulatum L. C. Rich & 28876 & $\mathrm{H}$ & Liparis nervosa (Thunb.) Lindl. & 28958 & $\mathrm{H}$ \\
\hline BROMELIACEAE & & & Paradisanthus mosenii Rchb. f. & ** & $\mathrm{H}$ \\
\hline Bilbergia sp & ** & $\mathrm{E}$ & Phymatidium myrtophillum Rodr. & 22848 & $\mathrm{E}$ \\
\hline CELASTRACEAE & & & POACEAE & & \\
\hline Maytenus robusta Reiss. & 21794 & $\mathrm{~T}$ & Poaceae sp1 & ** & $\mathrm{H}$ \\
\hline CLETHRACEAE & & & Poaceae sp2 & ** & $\mathrm{H}$ \\
\hline Clethra scabra Loisel & 21198 & $\mathrm{~T}$ & Poaceae sp3 & ** & $\mathrm{H}$ \\
\hline CLUSIACEAE & & & POLYPODIACEAE & & \\
\hline Clusia parviflora Humb. \& Bonpl. Ex Willd. & 23434 & $\mathrm{~T}$ & Microgramma vaccinifolia Copel. & 25852 & E \\
\hline COMMELINACEAE & & & Polypodium latipes Langsd. \& L.Fisch. & 25864 & $\mathrm{H}$ \\
\hline Dichorisandra thyrsiflora Mik. & 21212 & $\mathrm{H}$ & ROSACEAE & & \\
\hline CYPERACEAE & & & Prunus sellowii Koehne & 21500 & $\mathrm{~T} / \mathrm{S}$ \\
\hline Scleria secans (L.) Urb. & 28896 & $\mathrm{H}$ & RUBIACEAE & & \\
\hline DENNSTAEDIACEAE & & & Amaioua guianensis Aubl. & 22881 & $\mathrm{~S}$ \\
\hline Pteridium aquilinum Kuhn & ** & $\mathrm{H}$ & Diodia setigera DC. & $* *$ & $\mathrm{H}$ \\
\hline DILLENIACEAE & & & Lipostoma cf capitatum (Graham) D. Don & *** & $\mathrm{H}$ \\
\hline Davilla rugosa Poir. & 21193 & $\mathrm{~L}$ & Psychotria barbiflora DC. & 22484 & $\mathrm{~S}$ \\
\hline DIOSCOREACEAE & & & Rudgea villiflora $\mathrm{K}$. Schum. ex Standl. & 28965 & $\mathrm{~S}$ \\
\hline Dioscorea laxiflora Mart. ex Griseb. & ** & $\mathrm{L}$ & Rubiaceae 1 & ** & $\mathrm{S}$ \\
\hline Dioscorea $\mathrm{sp}$ & ** & $\mathrm{L}$ & RUTACEAE & & \\
\hline DRYOPTERIDACEAE & & & Zanthoxylum rhoifolium Lam. & $* *$ & $\mathrm{~T}$ \\
\hline Rumohra adiantiformis (Forst.) Ching & 28877 & $\mathrm{H}$ & SAPINDACEAE & & \\
\hline FABACEAE & & & Matayba guianensis Aubl. & 21494 & $\mathrm{~T}$ \\
\hline Andira anthelminthica (Vog.) Benth & 28902 & $\mathrm{~T}$ & SCHIZAEACEAE & & \\
\hline Desmodium adscendens (Sw.) DC. & 23920 & $\mathrm{H}$ & Schizaea pennula Sw. & 28878 & $\mathrm{H}$ \\
\hline Desmodium incanum DC. & 23917 & $\mathrm{H}$ & SMILACACEAE & & \\
\hline Fabaceae sp & ** & --- & Smilax campestris Griseb. & 23457 & $\mathrm{~L}$ \\
\hline ERYTROXYLACEAE & & & Smilax sp & ** & $\mathrm{L}$ \\
\hline Erytroxylum amplifolium (Mart.) Schulz & 21416 & $\mathrm{~T} / \mathrm{S}$ & SOLANACEAE & & \\
\hline EUPHORBIACEAE & & & Solanum pseudoquina A.St.Hil. & $* *$ & $\mathrm{~T}$ \\
\hline Alchornea triplinervia Müll.Arg. & 21408 & $\mathrm{~T}$ & SYMPLOCACEAE & & \\
\hline Pera glabrata (Schott) Poepp. ex Baill. & 21410 & $\mathrm{~T}$ & Symplocos phaeocladus (Mart.) A. DC. & 23011 & $\mathrm{~T}$ \\
\hline IRIDACEAE & & & Symplocos sp. & 28971 & $\mathrm{~T}$ \\
\hline Neomarica candida (Hassl.) Sprague & 21626 & $\mathrm{H}$ & THEACEAE & & \\
\hline LAURACEAE & & & Ternstroemia brasiliensis Camb. & 20880 & $\mathrm{~T} / \mathrm{S}$ \\
\hline Aniba firmula (Ness) Mez. & 20919 & $\mathrm{~T}$ & Gordonia fruticosa (Schrad.) H. Keng & $* *$ & $\mathrm{~T}$ \\
\hline Ocotea pulchella Mart. & 20902 & $\mathrm{~T} / \mathrm{S}$ & TRIGONACEAE & & \\
\hline Ocotea catharinensis $\mathrm{Mez}$ & 23486 & $\mathrm{~T}$ & Trigonia rotundifolia Nees & 28973 & $\mathrm{~L}$ \\
\hline Ocotea $\mathrm{sp}$ & ** & $\mathrm{T}$ & & & \\
\hline
\end{tabular}

---- habit not assigned due to inconclusive data;

** vegetative material - which was not accepted at UPCB. 
Table 2 - Species sampled in the structural analysis of an 8 year old fallow in the Lowland Atlantic Rain Forest, Itapoá, SC, Brazil; where $\mathrm{AD}$ - absolute density (individuals.ha ${ }^{-1}$ ); RD - relative density (\%); AF - absolute frenquency $(\%)$; RF - relative frequency $(\%)$; $\mathrm{ADO}$ - absolute dominance $\left(\mathrm{m}^{2} \mathrm{ha}^{-1}\right)$; RDO - relative dominance $(\%)$; IV - importance value; Ecol. group - ecological group; P - pioneer (large gap specialists); AP - anthropogenic pioneer; $\mathrm{O}$ - opportunist (small gap specialist); T - shade tolerant; * - values smaller than 0.001; ** - values smaller than 0.01. Some species were not included in any ecological group due to lack of - or inconclusive - data.

\begin{tabular}{|c|c|c|c|c|c|c|c|c|c|}
\hline & SPECIES & $\mathrm{AD}$ & $\mathrm{RD}$ & $\mathrm{AF}$ & RF & $\mathrm{ADO}$ & DOR & IV & Ecol. \\
\hline 1 & Psidium cattleianum & 1895.7 & 29.46 & 100.00 & 9.01 & 8.131 & 64.37 & 102.80 & $\overline{\mathrm{AP}}$ \\
\hline 2 & Eupatorium casarettoi & 2362.8 & 36.71 & 100.00 & 9.01 & 0.975 & 7.72 & 53.44 & $\mathrm{P}$ \\
\hline 3 & Ocotea pulchella & 1250.0 & 19.42 & 92.00 & 8.29 & 2.177 & 17.23 & 44.94 & AP \\
\hline 4 & Ternstroemia brasiliensis & 194.29 & 3.02 & 89.00 & 8.02 & 0.736 & 5.83 & 16.87 & AP \\
\hline 5 & Erythroxylum amplifolium & 125.71 & 1.95 & 67.00 & 6.04 & 0.080 & 0.63 & 8.62 & $\mathrm{AP}$ \\
\hline 6 & Myrsine coriacea & 104.29 & 1.62 & 69.00 & 6.22 & 0.021 & 0.17 & 8.01 & AP \\
\hline 7 & Guatteria australis & 75.71 & 1.18 & 53.00 & 4.77 & 0.070 & 0.55 & 6.50 & ค \\
\hline 8 & Andira anthelminthica & 27.14 & 0.42 & 33.00 & 2.97 & 0.172 & 1.36 & 4.75 & $\mathrm{O}$ \\
\hline 9 & Guapira opposita & 40.00 & 0.62 & 44.00 & 3.96 & 0.006 & 0.05 & 4.63 & $\mathrm{O}$ \\
\hline 10 & Eupatorium laevigatum & 31.43 & 0.49 & 39.00 & 3.51 & 0.002 & 0.01 & 4.01 & $P$ \\
\hline 11 & Syagrus romanzoffiana & 34.29 & 0.53 & 28.00 & 2.52 & 0.005 & 0.04 & 3.09 & $P$ \\
\hline 12 & Gomidesia fenzliana & 21.43 & 0.33 & 28.00 & 2.52 & 0.008 & 0.22 & 3.07 & $P$ \\
\hline 13 & Guapira asperula & 22.86 & 0.36 & 25.00 & 2.25 & 0.005 & 0.04 & 2.65 & $\mathrm{O}$ \\
\hline 14 & Clusia parviflora & 14.29 & 0.22 & 25.00 & 2.25 & 0.001 & 0.01 & 2.48 & $\mathrm{~T}$ \\
\hline 15 & Ilex pseudobuxus & 18.57 & 0.29 & 22.00 & 1.98 & 0.024 & 0.19 & 2.46 & $\mathrm{O}$ \\
\hline 16 & Gordonia fruticosa & 15.71 & 0.24 & 19.00 & 1.71 & 0.028 & 0.22 & 2.17 & $\mathrm{P}$ \\
\hline 17 & Baccharis cassinefolia & 22.86 & 0.36 & 19.00 & 1.71 & 0.003 & 0.02 & 2.09 & $\mathrm{O}$ \\
\hline 18 & Ilex integerrima & 11.43 & 0.18 & 17.00 & 1.53 & 0.004 & 0.03 & 1.74 & -- \\
\hline 19 & Ilex $s p$ & 11.43 & 0.18 & 17.00 & 1.53 & 0.002 & 0.02 & 1.73 & - \\
\hline 20 & Alchornea triplinervia & 11.43 & 0.18 & 14.00 & 1.26 & 0.015 & 0.12 & 1.56 & $\mathrm{O}$ \\
\hline 21 & Myrsine venosa & 12.86 & 0.20 & 14.00 & 1.26 & 0.004 & 0.04 & 1.50 & $\mathrm{~T}$ \\
\hline 22 & Ossaea amygdaloides & 11.43 & 0.18 & 14.00 & 1.26 & 0.001 & 0.01 & 1.45 & $\mathrm{P}$ \\
\hline 23 & Solanum pseudoquina & 7.14 & 0.11 & 14.00 & 1.26 & 0.004 & 0.03 & 1.40 & AP \\
\hline 24 & Ilex theezans & 7.14 & 0.11 & 14.00 & 1.26 & 0.003 & 0.03 & 1.40 & $\mathrm{O}$ \\
\hline 25 & Miconia sellowiana & 8.57 & 0.13 & 11.00 & 0.99 & 0.007 & 0.06 & 1.18 & $\mathrm{O}$ \\
\hline 26 & Pera glabrata & 8.57 & 0.13 & 11.00 & 0.99 & 0.006 & 0.05 & 1.17 & $\mathrm{O}$ \\
\hline 27 & Matayba guianensis & 5.71 & 0.09 & 11.00 & 0.99 & 0.001 & 0.01 & 1.09 & $\mathrm{O}$ \\
\hline 28 & Amaioua guianensis & 10.00 & 0.16 & 8.00 & 0.72 & 0.005 & 0.04 & 0.92 & $\mathrm{O}$ \\
\hline 29 & Symplocos phaeoclados & 4.29 & 0.07 & 8.00 & 0.72 & 0.006 & 0.05 & 0.84 & -- \\
\hline 30 & Symplocos $s p$ & 1.43 & 0.02 & 3.00 & 0.27 & 0.003 & 0.42 & 0.71 & -- \\
\hline 31 & Psychotria barbiflora & 10.00 & 0.16 & 6.00 & 0.54 & * & ** & 0.70 & $\mathrm{~T}$ \\
\hline 32 & Ossaea confertifolia & 4.29 & 0.07 & 6.00 & 0.54 & 0.001 & ** & 0.61 & $\mathrm{O}$ \\
\hline 33 & Maytenus robusta & 2.86 & 0.04 & 6.00 & 0.54 & 0.003 & 0.03 & 0.61 & $\mathrm{O}$ \\
\hline 34 & Rudgea villiflora & 2.86 & 0.04 & 6.00 & 0.54 & $*$ & $* *$ & 0.58 & $\mathrm{O}$ \\
\hline 35 & Ossaea brachystachia & 2.86 & 0.04 & 6.00 & 0.54 & $*$ & ** & 0.58 & $\mathrm{P}$ \\
\hline 36 & Myrtacea sp & 2.86 & 0.04 & 6.00 & 0.54 & $*$ & ** & 0.58 & -- \\
\hline 37 & Undetermined spl & 2.86 & 0.04 & 6.00 & 0.54 & $*$ & ** & 0.58 & ---- \\
\hline 38 & Miconia rigidiuscula & 2.86 & 0.04 & 6.00 & 0.54 & * & ** & 0.58 & $\mathrm{~T}$ \\
\hline 39 & Eupatorium inulaefolium & 2.86 & 0.04 & 6.00 & 0.54 & $*$ & $* *$ & 0.58 & $P$ \\
\hline 40 & Byrsonima ligustrifolia & 1.43 & 0.02 & 3.00 & 0.27 & 0.022 & 0.17 & 0.46 & $\mathrm{O}$ \\
\hline 41 & Eugenia umbelliflora & 1.43 & 0.02 & 3.00 & 0.27 & 0.015 & 0.12 & 0.41 & 1 \\
\hline 42 & Tibouchina clavata & 7.14 & 0.11 & 3.00 & 0.27 & 0.001 & 0.01 & 0.39 & $P$ \\
\hline 43 & Geonoma schottiana & 4.29 & 0.07 & 3.00 & 0.27 & 0.004 & 0.03 & 0.37 & $\mathrm{~T}$ \\
\hline 44 & Vernonia beyrichii & 2.86 & 0.04 & 3.00 & 0.27 & * & ** & 0.31 & $P$ \\
\hline 45 & Ocotea catharinensis & 1.43 & 0.02 & 3.00 & 0.27 & 0.002 & 0.01 & 0.30 & $\cdots$ \\
\hline 46 & Gomidesia palustris & 1.43 & 0.02 & 3.00 & 0.27 & 0.002 & 0.01 & 0.30 & P \\
\hline 47 & Ficus organensis & 1.43 & 0.02 & 3.00 & 0.27 & 0.001 & 0.01 & 0.30 & $\mathrm{O}$ \\
\hline 48 & Aniba firmula & 1.43 & 0.02 & 3.00 & 0.27 & 0.001 & 0.01 & 0.30 & $\mathrm{~T}$ \\
\hline 49 & Tabebuia chrysotricha & 1.43 & 0.02 & 3.00 & 0.27 & $*$ & ** & 0.29 & ---- \\
\hline 50 & Rubiaceae sp & 1.43 & 0.02 & 3.00 & 0.27 & $*$ & ** & 0.29 & -- \\
\hline 51 & Prunus sellowii & 1.43 & 0.02 & 3.00 & 0.27 & $*$ & ** & 0.29 & $\mathrm{O}$ \\
\hline 52 & Ocotea sp & 1.43 & 0.02 & 3.00 & 0.27 & * & ** & 0.29 & 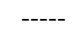 \\
\hline 53 & Myrcia multiflora & 1.43 & 0.02 & 3.00 & 0.27 & * & ** & 0.29 & $\mathrm{~T}$ \\
\hline 54 & Miconia cinerascens & 1.43 & 0.02 & 3.00 & 0.27 & $*$ & ** & 0.29 & $\mathrm{O}$ \\
\hline \multirow[t]{2}{*}{55} & Clethra scabra & 1.43 & 0.02 & 3.00 & 0.27 & $*$ & $* *$ & 0.29 & $\mathrm{O}$ \\
\hline & TOTAL & 6435 & 100 & 1110 & 100 & 12.632 & 100 & 300 & \\
\hline
\end{tabular}




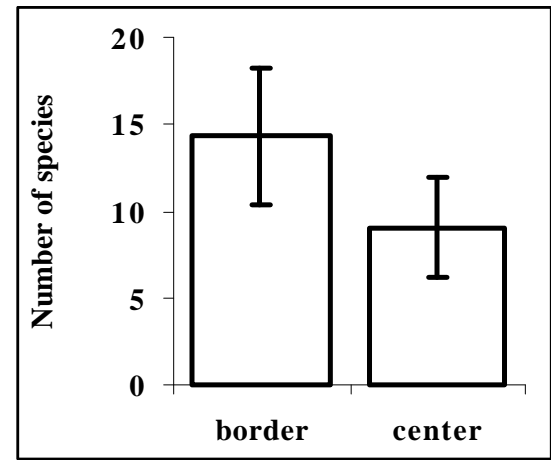

Figure 2 - Mean number of species in border and center plots (mean \pm one SD) in an 8-year-old fallow in the Lowland Atlantic Rain Forest. Itapoá. SC. Brazil.

Some of the species that occurred preferentially closer to the border were Miconia sellowia, Clethra scabra, and Pera glabrata. which all were observed occurring in small gaps inside the forest too (personal observation).

\section{DISCUSSION}

As reported to other initial successional stages, resulting from anthropogenic perturbation (i.e. Gomez-Pompa et al., 1991; Maser, 1994; Rosenzweig, 1995), the studied area showed a low diversity. The high diversity of Myrtaceae, Asteraceae, Melastomataceae and Rubiaceae was also expected since these are well-represented families in tropical and subtropical South America (Barroso, 1984, 1986; Cronquist, 1988). On the other hand, the low diversity of Orchidaceae, reported as a very speciouse family of the Atlantic Rain Forest, could be due to the small number of trees with girth big enough to allow the profusion of epiphytes.

Based on geographical distribution data, one could predict the occurrence of those very speciouse genuses found in the studied area. Ilex (Aquifoliaceae) is a genus that has its center of distribution in mid-southern South America (Edwin and Reitz, 1967). Eupatorium (Asteraceae) and Ocotea (Lauraceae) have beenn reported as pantropical with highest diversity in South America (Cabrera and Klein, 1989; Vattimo, 1956) as well Miconia (Melastomataceae) and Gomidesia (Myrtaceae), both neotropical (Wurdack, 1962; Legrand and Klein, 1967). Ossaea (Melastomataceae) is the only one cited as a genus with higher diversity in Asia (Wurdack, 1962).

The considerable similarity among the studied site and Restinga Vegetation could be the result of similarities in climate, soil and the regional geological and species' evolutionary history (Pianka, 1994; Brown, 1995; Gentry, 1982; Futuyma, 1992) which created a gradual transition from Restinga to Atlantic Rain Forest. Apparently, this similarity might be due to (1) both kinds of vegetation grow on Podzol and quartzsands soils and (2) theoretically, over hundreds or thousands of years, Restinga Vegetation could have become Lowland Atlantic Rain Forest through the action of the biota on the evolution of soils. So, these two kinds of vegetation are somewhat related on a greater spatio-time scale.

It is important, when discussing similarity, to bear in mind that even though the successional process is known, stochastic factors have strong effects on an establishing community's composition (Mabberley, 1992), in a way that it makes each community unique for each area. This was clear when we found that the closest area to the present study - an adjacent 35-year-old fallow - was only $43.7 \%$ similar. It was interesting to notice an increase in the similarity with this 35-year-old fallow as we calculated it for greater minimum $\mathrm{DBH}$. With higher minimum girth, the probability of including just the oldest individuals was greater - they were the first to establish when the area had just been abandoned. Therefore, the increase in the similarity with the increase of minimum girth showed that the first species to colonize the area studied by Lolis (1996) were similar to the ones colonizing the area of the present study; which showed some trend in predictability of the species colonizing the area after abandonment.

The impact of clear cutting and fire activities certainly have a strong influence in this young community's structure, i.e., P. cattleianum, $T$. brasiliensis and E. amplifolium have most of their individuals with more than oone stem (35 stems in some $P$. cattleianum). Probably the successive clear cuts associated with the ability of resprouting from roots and/or stems might have been the cause of the increase in density of these species.

The species with the highest density, Eupatorium casarettoi, is a shrub typical of dunes close to the beach and of open Restinga Vegetation (Cabrera and Klein, 1989). This was probably because of the proximity of the Reserve to the beach $-5 \mathrm{~km}$. Since its dispersal is anemochorous and there are long open areas due to real estate expansion, this species appears further into the plain. 
Table 3 - Sørensen’s similarity indices (IS $S_{S}$ ) obtained comparing floristic data from the present study site and from other different Atlantic Forest and Restinga sites.

\begin{tabular}{|c|c|c|c|}
\hline STUDY SITE & CRITERIA & AUTHOR & $\overline{\mathrm{IS}_{\mathrm{S}}}$ \\
\hline LARF, Itapoá, SC; 35 year old fallow & $\mathrm{DBH} \geq 10 \mathrm{~cm}$ & Lolis (1996) & 43.67 \\
\hline LARF, Itapoá, SC; 35 year old fallow & $\mathrm{DBH}<5 \mathrm{~cm}$ & Lolis (1996) & 43.66 \\
\hline LARF, Itapoá, SC; 35 year old fallow & $\mathrm{DBH} \geq 8 \mathrm{~cm}$ & Lolis (1996) & 42.1 \\
\hline LARF, Itapoá, SC; 35 year old fallow & $\mathrm{DBH} \geq 5 \mathrm{~cm}$ & Lolis (1996) & 40.33 \\
\hline Restinga Forest, Ilha do Mel, Paranaguá-PR & $\mathrm{DBH} \geq 5 \mathrm{~cm}$ & Silva (1990) & 34.86 \\
\hline Restinga Forest, Ilha do Cardoso, SP & $\mathrm{DBH} \geq 1.6 \mathrm{~cm}$ & Sugiyama (1993) & 29.6 \\
\hline SMARF, Guaraqueçaba, PR & $\mathrm{PBH} \geq 10 \mathrm{~cm}$ & Athayde (Com.Pes.) & 28.28 \\
\hline LARF, Itapoá SC, intact forest & $\mathrm{DBH}<5 \mathrm{~cm}$ & Negrelle (1995) & 27.58 \\
\hline SMARF, 13 year old fallow, Morretes, PR & $\mathrm{DBH} \geq 6.4 \mathrm{~cm}$ & Guapyassu (1994) & 17.02 \\
\hline Restinga Forest, Emboaba, Osório, RS & $\mathrm{DBH} \geq 5 \mathrm{~cm}$ & Dillenburg (1986) & 15.15 \\
\hline LARF, Morretes, PR & $\mathrm{PBH} \geq 20 \mathrm{~cm}$ & Silva (1985) & 13.59 \\
\hline Caxetais, coastal forested marshes & $\mathrm{DBH} \geq 10 \mathrm{~cm}$ & Ziller (1992) & 11.96 \\
\hline SMARF, 15 year old fallow, Vale do Ribeira, SP & Height $>1 \mathrm{~m}$ & Torezan (1995) & 9.41 \\
\hline
\end{tabular}

LARF - Lowland Atlantic Rain Forest SMARF- Submontane Atlantic Forest; DBH - diameter at breast height; $\mathrm{PBH}$ - perimeter at breast height.

The categorization of species in ecological groups, although a very good tool, it is only didactic, since in nature there is a continuum of degree of dependence on gaps for regeneration. Besides, many times it is applicable only for the region where the research is developed, because environmental pressures upon gene regulation and expression mechanisms may alter the structure and physiologic behavior of the individuals from a population in a small period of time (Cronquist, 1988; Futuyma, 1992; Wolf, 1995). Therefore, the results revealed here might not be in perfect agreement with the categorization of the same species elsewhere.

The spatial distribution within the clearing may be due to the existence of less extreme conditions closer to the border when compared with its center - which receives more light - has higher soil surface temperature and lower relative humidity (Popma et al., 1988; Matlack, 1994; Denslow, 1987). Therefore, spatial heterogeneity leads to differential colonization of species in different parts of a clearing in the forest (Turner et al., 1998). In fact, some authors have found preferential establishment of some species. i.e., Miconia argentea in small gaps, in the border of big gaps, or in center of big gaps only after these have been colonized by pioneers, where/when ambient conditions are less extreme (Brandani et al., 1988; Brokaw, 1987; Denslow, 1987). We must also consider the fact that many species do not have very efficient dispersal mechanisms. So the distance from the source of diaspores will affect the amount (Yao et al., 1999) and the diversity of the seed rain falling at the clearing the closer to the border, the greater and richer the seed rain will be (Garwood, 1989).

From the results obtained in this study, we can hypothesize that succession in these anthropogenic clearings takes place in the following pattern: (1) pioneer and anthropogenic pioneer species first colonize the area abandoned; (2) after this first 'step', the environmental conditions begin to change and then small gap specialists and tolerant species start to colonize the area too - mainly on the borders of the opening. In such pattern, we could expect a gradual increase in species diversity over time.

It is clear that the geological formation of these Quaternary plains is the main cause of this vegetation's low plant diversity and of its similarity with Restinga Vegetation.

In regard to the previsibility of the species composition in fallows through time, we observed that in large anthropogenic perturbations, like in the present area, it was more likely to find certain species more frequently than others, where there was no soil variation. In small natural gaps this was not the case because these areas are rapidly colonized and stochastic factors seem to play a major role in its composition, reducing its previsibility (Foster and Tilman, 2000). We also found that in these anthropogenic clearings, species other than the usual pioneers dominated the colonization process, since the environmental pressures in these areas were also different.

Attention must be drawn to the fact that most part of this type of coastal ecosystems are being turned 
into pastures, Pinus plantations and real estate. We do not know the functions of these ecosystems on regional climate, hydrology and nutrient cycling. Therefore, we need better understanding of the composition and functioning of regenerating plant communities.

\section{ACKNOWLEDGMENTS}

We thank Alexandre Lorenzeto and Melissa Browning for the field work; the grant given by CAPES for Cleber Salimon; the staff of the Universidade Federal do Paraná., Pr. Brazil; Mr Jorge for his empirical knowledge during field work. and the Machado family - the owners of the reserve.

\section{RESUMO}

A maior parte das áreas florestais no domínio da Floresta Atlântica se encontra degradada devido a diferentes pressões antrópicas. No intuito de ampliar os conhecimentos sobre relictos de florestas nativas intactas, e também de áreas abandonadas para se obter dados sobre os processos naturais de regeneração, foi realizado um estudo da composição florística, estrutura e dinâmica de uma comunidade vegetal em estágio seral inicial de 8 anos. em Floresta Ombrófila Densa das Terras Baixas, na Reserva Volta Velha, Itapoa-SC, Brasil. Foram utilizados os métodos usuais de coleta, herborização e identificação das espécies encontradas, e a análise estrutural foi feita utilizando-se 36 parcelas retangulares de $20 \mathrm{X}$ $10 \mathrm{~m}$, sendo incluídas todas as plantas arbustivo/arbóreas com no mínimo 1 metro de altura. Os resultados obtidos foram os seguintes: 1- Foram encontradas 96 espécies, dentro de 68 gêneros e 44 famílias; as famílias com maior número de espécies foram Myrtaceae e Asteraceae com 8 espécies cada, e o gênero mais representado foi Ilex, com 4 espécies; 2- As espécies mais importantes (parâmetros fitossociológicos) no local foram Psidium cattleianum, Eupatorium casarettoi, Ocotea pulchella e Ternstroemia brasiliensis; 4- A área mais similar à do presente estudo foi uma área vizinha abandonada há 35 anos; áreas de restinga também demostraram ser relativamente semelhantes; 5- O grupo ecológico com maior número de espécies foi $\mathrm{o}$ de oportunistas (especialistas de pequenas clareiras), e houve um maior número de espécies nas parcelas de borda, mostrando que a maior parte das espécies não toleram as condições extremas do centro da clareira. e sua colonização se dá via bordas.

\section{REFERENCES}

Ab'saber, A. N. (1979), Os mecanismos da desintegração das paisagens tropicais no Pleistoceno Efeitos paleoclimáticos do período Würm-Wisconsin no Brasil. Inter-Facies - escritos e documentos. UNESP/IBILCE. 4.

Barroso, G. M. (1984), Sistemática de Angiospermas do Brasil, 2, Imprensa Universitária da Universidade de Viçosa, Viçosa. MG.

Barroso, G. M. (1986), Sistemática de Angiospermas do Brasil. 3, Imprensa Universitária da Universidade de Viçosa, Viçosa, MG.

Brandani, A.; Hartshorn, G. and Orians, H. (1988), Internal heterogeneity of gaps and species richness in Costa Rican tropical wet forest. Trop. Ecology, 99-119

Brokaw, N. V. L. (1987), Gap-phase regeneration of three pioneer tree species in a Tropical Forest. Jour. of Ecology, 75, 9-19

Brown, J. H. (1995), Macroecology. The University of Chicago Press, London.

Cabrera, A. L. and Klein, R. M. (1989), Compostas 4Tribo: Eupatorieae. In: Reitz, R. Flora Ilustrada Catarinense. Itajaí, SC.

Cronquist, A. (1988), The evolution and classification of flowering plants. The New York Botanical Garden, Bronx, NY.

Denslow, J. S. (1980), Gap partitioning among Tropical Rain Forest trees. Tropical Succession, 47-55

Denslow, J. S. (1987), Tropical rainforest gaps and tree species diversity. Ann Rev Eco Syst, 18, 431-451

Dillenburg, L. R. (1986), Estudo Fitossociológico do Estrato Arbório da Mata Arenosa de Restinga de Emboaba, Osório, RS, Porto Alegre, dessertação mestrado. Universidade Federal do Rio Grande do Sul.

Dorneles, L. P. P. (1996), Florística e estrutura do componente Herbáceo e Aspectos da Regeneração Natural de Espécies Arbóreas, de um Estágio Seral da Floresta Atlântica na Reserva de Volta Velha, Mun. Itapoá - SC, Curitiba, dissertação de mestrado. Universidade Federal do Paraná.

Edwin, G. and Reitz, P. R. (1967), Aquifoliáceas. In: REITZ, P. R. Flora Ilustrada Catarinense. Itajaí, SC. 
Foster, B. L. and Tilman, D. (2000), Dynamic and static views of succession: Testing the descriptive power of the chronosequence approach. Plant Ecology, 146(1), $1-10$

Futuyma, D. J. (1992), Biologia Evolutiva. Sociedade Brasileira de Genética, Brasil.

Garwwod, N. C. (1989), Tropical Soil Seed Banks: a Review. In: Leck, Parker, Sympson. Ecology of Soil Seed Banks. Academic Press, 149-209

Gentry, A. H. (1982), Neotropical Floristic Diversity: phytogeographical coennections between Central and South America, Pleistocene climatic flutuations or an accident of the Andian Orogeny? Annals of the Missouri Botanical Garden, 69, 557-593

Gomez-Pompa, A.; Whitmoe, T. C. and Hadley, M. (1991), Rain Forest Regeneration and Management. Man and the Biosphere Series 6. UNESCO and the Parthenon Publishing Group, Paris.

Guapyassu, M. S. (1994), Caracterização fitossociológica de três fases sucessionais de uma Floresta ombrófila Densa Submontana, Morretes-PR, Curitiba, dissertação de mestrado. Universidade Federal do Paraná.

Klein, R. M. (1978), Mapa Fitogeográfico do Estado de Santa Catarina. In: Reitz, P. R. Flora Ilustrada Catarinense, HBR. Itajaí. SC. $24 \mathrm{p}$

Legrand, D. and Klein, R. M. (1967), Mirtáceas 1. In: Reitz, P. R. Flora Ilustrada Catarinense. Itajaí, SC.

Lolis, S. (1996), Análise Fitossociológica de um Estágio Seral de Floresta Ombrófila das Terras Baixas, Reserva de Volta Velha - Itapoá - SC. Curitiba, dissertação de mestrado. Universidade Federal do Paraná.

Mabberley, D. J. (1992), Tropical Rain Forest Ecology. Blackie, Glasgow.

Magurran, A. E. (1988), Diversidad ecologica y su medicion. Ediciones Vedra. Barcelona.

Maser, C. (1994), Sustainable Forestry: Filosophy, Science and Economics. St. Lucie Press, Delray Beach. FL.

Matlack, G. R. (1994), Vegetation dynamics of the forest edge - trends in space and successional time. Journal of Ecolgy, 82(1), 113-124

Mueller-Dombois, D. and Ellemberg, H. A. (1974), Aims and methods of vegetation ecology. New York. John Wyley. 547p

Negrelle, R. R. B. (1995), Composição florística, estrutura fitossociológica e dinâmica de regeneração da Floresta Atlântica da Reserva de Volta Velha, Mun. Itapoá, SC._São Carlos, tese (doutorado em ecologia). Universidade Federal de São Carlos-SP

Pianka, E. R. (1994), Evolutionary ecology. Harper Collins College Publishers. New York, NY

Popma, J.; Bongers, F.; Martinez-Ramos, M. and Veneklass, E. (1988), Pioneer species distribuition in tree fall gaps in Neotropical Rainforest; a gap definition and its consequences. Jour. of Trop. Ecology, 4, 77-88
Por, F. D. (1992), Sooretama the Atlantic Rain Forest of Brazil. The Hague: SPB Academic. 130p

Rosenzweig, M. L. (1995), Species diversity in space ad time. Cambridge University Press.

Santa Catarina, (1986), Gabinete de planejamento e coordenação geral. Sub-chefia de Estatística, Geografia e Informática. Atlas de Santa Catarina. Rio de Janeiro. Aerofoto Cruzeiro.

Silva, C. S. (1985), Composição florística e estrutura fitossociológica da Floresta Tropical Ombrófila da Enconsta Atlântica no Município de Morretes Estado do Paraná. Curitiba, dissertação de mestrado. Universidade Federal do Paraná.

Silva, S. M. (1990), Composição florística e fitossociológica de um trecho de Floresta de Restinga na Ilha do Mel, Paranaguá, PR. Campinas, dissertação de mestrado, Universidade Estadual de Campinas, SP.

Sugyiama, M. and Mantovani, W. (1993), Fitossociologia de um trecho de Mata de Restinga na Ilha do Cardoso. SP. In: III Simpósio de Ecossistemas da Costa Brasileira. ACIESP, 49-57

Torezan, J. M. D. (1995), Estudo da sucessão secundária na floresta ombrófila densa sub-montana, em áreas anteriormente cultivadas pelo sistema de "coivara" em Iporanga, SP. Curitiba. Dissertação de mestrado. UFPR. 89p

Turner, M. G.; Baker, W. L.; Peterson, C. J. and Peet, R. K. (1998), Factors influencing succession: Lessons from large, infrequent natural disturbances. Ecosystems 1(6), 511-523

Vattimo, I. (1956), O gênero Ocotea Aubl. no Sul do Brasil. Rodriguésia, 30-31, 265-350

Whitmore, T. C. (1989), Canopy gaps and the two major groups of forest trees. Ecology, 70(3), 536-538

Wolf, U. (1995), The genetic contribuition to the phenotype. Hum. Genet., 95, 127-148

Wurdack, J. J. (1962), Melastomataceae of Santa Catarina. Sellowia, (14), 109-208

Yao, J.; Holt, R. D.; Rich, P.M. and Marshall, W. S., (1999), Woody plant colonization in an experimentally fragmented landscape. Ecography 22(6), 715-728

Ziller, S. R. (1992), Análise Fitossociológica de Caxetais. Curitiba, dissertação de mestrado, Universidade Federal do Paraná.

Received: July 19, 1999; Revised: September 21, 1999; Accepted: August 16; 2000. 